\title{
Magnetic susceptibility of an integrable anisotropic spin ladder system
}

\author{
A. P. Tonel ${ }^{1}$, S. R. Dahmen ${ }^{1}$, A. Foerster ${ }^{1}$ and A. L. Malvezzi ${ }^{2}$ \\ ${ }^{1}$ Instituto de Física da UFRGS \\ Av. Bento Gonçalves 9500, Porto Alegre, RS - Brazil \\ ${ }^{2}$ Departamento de Física da UNESP \\ Av. Eng. Luiz Edmundo Carrijo Coube, s/n, Bauru, SP-Brazil
}

August 31, 2018

\begin{abstract}
We investigate the thermodynamics of an integrable spin ladder model which possesses a free parameter besides the rung and leg couplings. This model is exactly solvable by means of the Bethe Ansatz and exhibits a phase transition between a gapped and a gapless spin excitation spectrum. The magnetic susceptibility is obtained numerically and its dependence on the anisotropy parameter is determined. A comparison between the spin gap obtained from the susceptibility curve and the one obtained from the Bethe Ansatz Equations is made and a good agreement found. A connection with the compounds $\mathrm{KCuCl}_{3}$ [1, 2, $\mathrm{Cu}_{2}\left(\mathrm{C}_{5} \mathrm{H}_{12} \mathrm{~N}_{2}\right)_{2} \mathrm{Cl}_{4}, 3,4,5$, and $\left(\mathrm{C}_{5} \mathrm{H}_{12} \mathrm{~N}\right)_{2} \mathrm{CuBr}_{4}$ [6] in the strong coupling regime is made and our results for the magnetic susceptibility fit the experimental data remarkably well.
\end{abstract}

PACS: 75.10.Jm, 71.10.Fd, 03.65.Fd 


\section{Introduction}

The study of spin ladders, both from an experimental as well as from a theoretical point of view has gained much attention in the last few years and has by now a literature of its own. On the one hand with the discovery of high-Tc superconductivity in doped cuprate materials [7, a tremendous effort has been made to understand the physics of these compounds, in which the $\mathrm{Cu}-\mathrm{O}$ ions build up a two-dimensional lattice. This task is made more difficult by the lack of exactly integrable models as compared to 1-D where an abundance of such models has been known for decades. On the other hand spin ladders are prototypes of quasi one-dimensional systems and as such represent an excellent proving ground for studying the transition from the more amenable and better understood physics of one-dimensional quantum systems to two-dimensional behavior. They exhibit antiferromagnetic (AF) ground states, where at each site of the lattice (in the undoped state) an electron occupies one of two spin states [8]. As such they can be reasonably well approximated by the archetypical AF Heisenberg model or some suitable generalization thereof. In one dimension the Heisenberg model is exactly solvable via the Bethe Ansatz and from the solution it is known that in the AF regime, up to a given threshold of the spin exchange interaction, the elementary spin excitations are gapless (beyond the threshold one has a Néel-like groundstate with gapped excitations) [9, 10]. The existence of a spin gap is critical for the occurence of superconductivity under doping, whereby the holes introduced through doping undergo a Bose-Einstein condensation. By introducing the concept of a ladder model this apparent contradiction is resolved, since these systems allow for the formation of singlet states along the rungs which in turn are responsible for the appearance of a spin gap. (Strictly speaking the singlet states can only form when there is an even number of legs [11,12]). Recently, with the rapid progress being made in nano-engineering, many different species of ladder compounds such as $\mathrm{S}_{r} \mathrm{Cu}_{2} \mathrm{O}_{3}, \mathrm{La}_{1-x} \mathrm{Sr}_{x} \mathrm{CuO}_{2.5}, \mathrm{Sr}_{14-x} \mathrm{Ca}_{x} \mathrm{Cu}_{24} \mathrm{O}_{41}$ and other organic compounds [2] have been investigated in this context. This theory is now supported by a substantial body of experimental evidence.

However, in contradistinction to its one-dimensional counterpart, the two-dimensional Heisenberg ladder model cannot be solved exactly. So in the search for exactly integrable models which might give one a better insight into the physics of ladder systems, many authors have considered generalized models which incorporate additional interaction terms without violating integrability $13,14,15,16,17,18,19,20,21,22,23,24$. Notwithstanding the fact that these models exhibit realistic physical properties such as the existence of a spin gap [24,25] and the magnetization plateaus at fractional values of the total magnetization [26], up to our knowledge none has been used to predict physical quantities that could be compared directly with experimental data.

The main goal of the present work is to show that integrable ladder models can be used to model experimental data. In particular, we discuss a generalized integrable spin ladder with incorporates an additional anisotropy parameter besides the rung and leg interactions and show that the magnetic susceptibility as obtained from this model as a function of temperature fits well the experimental data for the ladder compounds $\mathrm{KCuCl}_{3}$, 1, 2, $\mathrm{Cu}_{2}\left(\mathrm{C}_{5} \mathrm{H}_{12} \mathrm{~N}_{2}\right)_{2} \mathrm{Cl}_{4}$ [3, 4, 5] and $\left(\mathrm{C}_{5} \mathrm{H}_{12} \mathrm{~N}\right)_{2} \mathrm{CuBr}_{4}$ [6]. The model is exactly solvable by the Bethe Ansatz and reduces to the model introduced by Wang [24,25] for some special 
limit of this extra parameter. This additional free parameter, which we call $t$, brings about an anisotropy-dependent critical line through an explicit functional dependence of the excitation gap. The thermodynamics of the model is investigated and the magnetic susceptibility curve as a function of the temperature is obtained. The influence of this anisotropy parameter on the thermodynamics of the model is also determined. A comparison between the spin gap obtained from the susceptibility curve and that one obtained from the Bethe ansatz equations is performed and a good agreement is found.

The paper is divided as follows: In section 2 we describe the model and its Bethe Ansatz solution. Section 3 discusses the thermodynamical properties of the model and is also devoted to a comparison between the experimental results and our findings. We conclude the paper with a brief summary.

\section{The model}

Let us begin by presenting the integrable anisotropic spin ladder model in the presence of an external magnetic field $h$ [23], whose Hamiltonian reads

$$
H=\sum_{j=1}^{L}\left[J_{l} h_{j, j+1}+\frac{J_{r}}{2}\left(\overrightarrow{\sigma_{j}} \cdot \overrightarrow{\tau_{j}}-1\right)-\frac{h}{2}\left(\sigma_{j}^{z}+\tau_{j}^{z}\right)\right]
$$

where

$$
\begin{aligned}
h_{j, j+1} & =\frac{1}{4}\left(1+\sigma_{j}^{z} \sigma_{j+1}^{z}\right)\left(1+\tau_{j}^{z} \tau_{j+1}^{z}\right)+\left(\sigma_{j}^{+} \sigma_{j+1}^{-}+\sigma_{j}^{-} \sigma_{j+1}^{+}\right)\left(\tau_{j}^{+} \tau_{j+1}^{-}+\tau_{j}^{-} \tau_{j+1}^{+}\right) \\
& +\frac{1}{2}\left(1+\sigma_{j}^{z} \sigma_{j+1}^{z}\right)\left(t^{-1} \tau_{j}^{+} \tau_{j+1}^{-}+t \tau_{j}^{-} \tau_{j+1}^{+}\right)+\frac{1}{2}\left(t^{-1} \sigma_{j}^{+} \sigma_{j+1}^{-}+t \sigma_{j}^{-} \sigma_{j+1}^{+}\right)\left(1+\tau_{j}^{z} \tau_{j+1}^{z}\right) .
\end{aligned}
$$

Periodic boundary conditions are imposed along the legs. As usual $\overrightarrow{\sigma_{j}}$ and $\overrightarrow{\tau_{j}}$ are Pauli matrices acting on site $j$ of the upper and lower legs respectively, $J_{r}\left(J_{l}\right)$ is the strength of the rung coupling (leg coupling) and $t$ is a free parameter which introduces an anisotropy in the leg and in the interchain interactions. Throughout this paper $L$ stands for the number of rungs (the length of the ladder). By setting $t \rightarrow 1$ in Eq. (11) one recovers the isotropic model of Wang [25] based on the $S U(4)$ symmetry (strictly speaking Wang's model is $S U(4)$-invariant only in the absence of the rung interactions). The Hamiltonian is invariant under the interchange of the legs, i.e. $\overrightarrow{\sigma_{j}} \leftrightarrow \overrightarrow{\tau_{j}}$. Moreover, under spin inversion

on both leg spaces it is also invariant under the exchange $t \leftrightarrow t^{-1}$. The energy eigenvalues of (11) are given by

$$
\frac{E}{J_{l}}=-\sum_{j=1}^{M_{1}}\left(\frac{1}{\lambda_{j}^{2}+1 / 4}-2 \frac{J_{r}}{J_{l}}+\frac{h}{J_{l}}\right)+\left(1-2 \frac{J_{r}}{J_{l}}\right) L+\frac{h}{J_{l}}\left(M_{2}+M_{3}\right)
$$

where the $\lambda_{j}$ 's are solutions of the three-level nested Bethe ansatz equations

$$
t^{\left(L-2 M_{3}\right)}\left(\frac{\lambda_{j}-i / 2}{\lambda_{j}+i / 2}\right)^{L}=\prod_{l \neq j}^{M_{1}} \frac{\lambda_{j}-\lambda_{l}-i}{\lambda_{j}-\lambda_{l}+i} \prod_{\alpha=1}^{M_{2}} \frac{\lambda_{j}-\mu_{\alpha}+i / 2}{\lambda_{j}-\mu_{\alpha}-i / 2}
$$




$$
\begin{aligned}
t^{\left(L-2 M_{3}\right)} \prod_{\beta \neq \alpha}^{M_{2}} \frac{\mu_{\alpha}-\mu_{\beta}-i}{\mu_{\alpha}-\mu_{\beta}+i} & =\prod_{j=1}^{M_{1}} \frac{\mu_{\alpha}-\lambda_{j}-i / 2}{\mu_{\alpha}-\lambda_{j}+i / 2} \prod_{\delta=1}^{M_{3}} \frac{\mu_{\alpha}-\nu_{\delta}-i / 2}{\mu_{\alpha}-\nu_{\delta}+i / 2} \\
t^{\left(L+2 M_{2}-2 M_{1}\right)} \prod_{\gamma \neq \delta}^{M_{3}} \frac{\nu_{\delta}-\nu_{\gamma}-i}{\nu_{\delta}-\nu_{\gamma}+i} & =\prod_{\alpha=1}^{M_{2}} \frac{\nu_{\delta}-\mu_{\alpha}-i / 2}{\nu_{\delta}-\mu_{\alpha}+i / 2}
\end{aligned}
$$

For the sake of convenience we take from here on $J_{l}=1$. From an analysis of these equations it follows that for $J_{r}>1+\frac{1}{2}\left(t+\frac{1}{t}\right)$ the reference state becomes the ground state and any excitation is gapped. In this region the ground state is given by a product of rung singlets, indicating a dimerization along the rungs. The energy gap $\Delta$ can be calculated using the exact Bethe ansatz solution and has the form

$$
\Delta=2\left(J_{r}-1-\frac{h}{2}-\frac{1}{2}\left(t+\frac{1}{t}\right)\right)
$$

By solving $\Delta=0$ for $J_{r}$ we find the critical value $J_{r}{ }^{c}=1+\frac{1}{2}\left(t+\frac{1}{t}\right)$, indicating the critical line at which the quantum phase transition from the dimerized phase to the gapless phase occurs. This result is depicted in Fig. 1.

Notice that by choosing $t$ real one has ${J_{r}}^{c} \geq 2$ which corresponds to the strong coupling limit. We therefore expect our anisotropic model to be a good candidate for describing some organic ladder compounds, such as $\mathrm{KCuCl}_{3}, \mathrm{Cu}_{2}\left(\mathrm{C}_{5} \mathrm{H}_{12} \mathrm{~N}_{2}\right)_{2} \mathrm{Cl}_{4}, \mathrm{TlCuCl} \mathrm{l}_{3}$ and $\left(\mathrm{C}_{5} \mathrm{H}_{12} \mathrm{~N}\right)_{2} \mathrm{CuBr} r_{4}$.

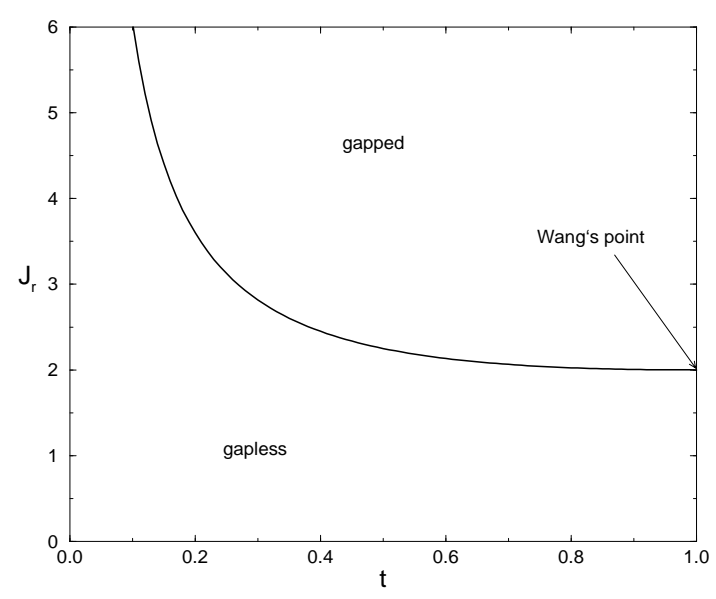

Figure 1: The phase diagram of (1) in the $\left(J_{r} \times t\right)$-plane in units of $J_{l}$. The arrow indicates Wang's point $(\mathrm{t}=1)$. The curve $J_{r}{ }^{c}=1+(t+1 / t) / 2$ divides the gaped from the gapless phase.

\section{Comparison with experimental data}

Our main goal is to calculate the magnetic susceptibility $\chi$ of our exactly integrable ladder Hamiltonian and compare it to experimental data on the compounds mentioned before. 
In order to do this we block-diagonalize the Hamiltonian Eq. (1) for a sequence of finite chains (up to 6 rungs or 12 sites) and for different values of the coupling $J_{r}$ and anisotropy $t$. With these spectra we generate a sequence of finite-size susceptibilities $\chi^{(\mathrm{L})}$ as a function of the temperature $T$ and then apply a standard Bulirsch-Stoer acceleration technique [27. to the sequence in order to determine the susceptibility in the thermodynamic limit $L \rightarrow \infty$. An example for $J_{r}=7$ and $t=1$ is presented in Fig. 2. In order to compare

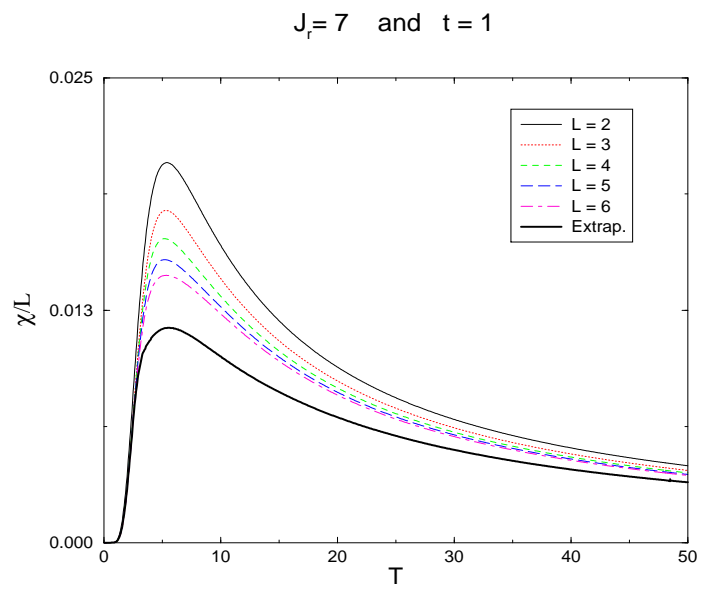

Figure 2: The finite-size susceptibility per site for the case $J_{r}=7$ and $t=1$ for varying number of rungs $L$ and the extrapolated curve.

our results with experimental data available on a series of compounds, a few remarks on the behavior of the susceptibility as a function of $J_{r}$ and $t$ are necessary, as well as the procedure used for choosing the values of our parameters. First we observe that for a fixed value of $t$ there is a 'smoothing out' of the susceptibility curve for increasing $J_{r}$ while for fixed $J_{r}$ the magnetic susceptibility increases with decreasing $t$, as can be seen in Fig. $3 a$. The second point is that in all cases the susceptibility presents an exponential decay for low temperature $T<<\Delta^{*}$ where $\Delta^{*}$ is the spin gap of the system

$$
\chi \propto \frac{e^{-\frac{\Delta^{*}}{T}}}{\sqrt{T}}
$$

which is in agreement with the result of Troyer et. al. 28] for the Heisenberg spin ladder model. By linearizing Eq. (5) a numerical value for the spin gap $\left(\Delta^{*}\right)$ can be found. When compared to the exact expression of the spin gap $\Delta$ obtained from the Bethe Ansatz Equations (44) at $T=0$ an excellent agreement is found as can be seen in Table 1. The linearized curves are depicted in Fig. 3b.

We remark that the behavior of the magnetic susceptibility for the critical value of the couplings $\left(J_{r}^{c}\right)$ is of the form

$$
\chi \sim \frac{1}{\sqrt{T}}
$$

which indicates a typical quantum critical behavior. This had already been predicted in [24, 25] for the isotropic case as illustrated in Fig. 4 for different values of $t$. By 
(a)
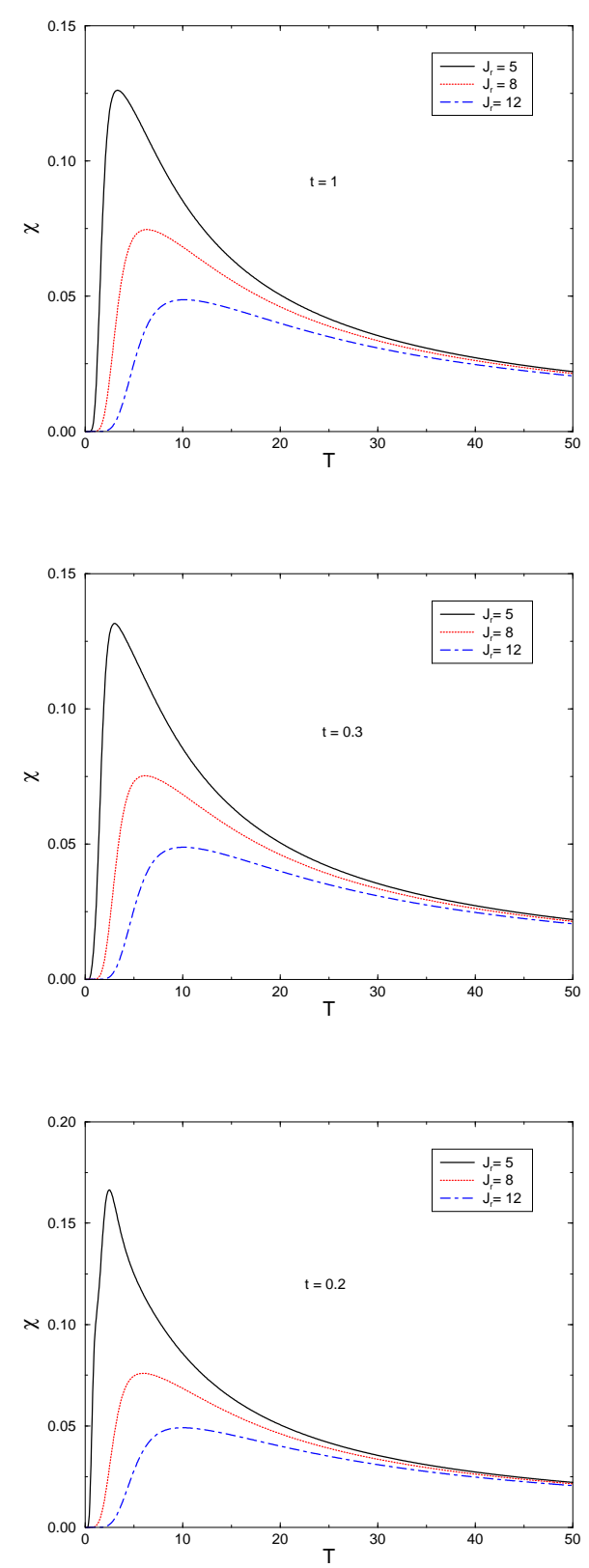

(b)
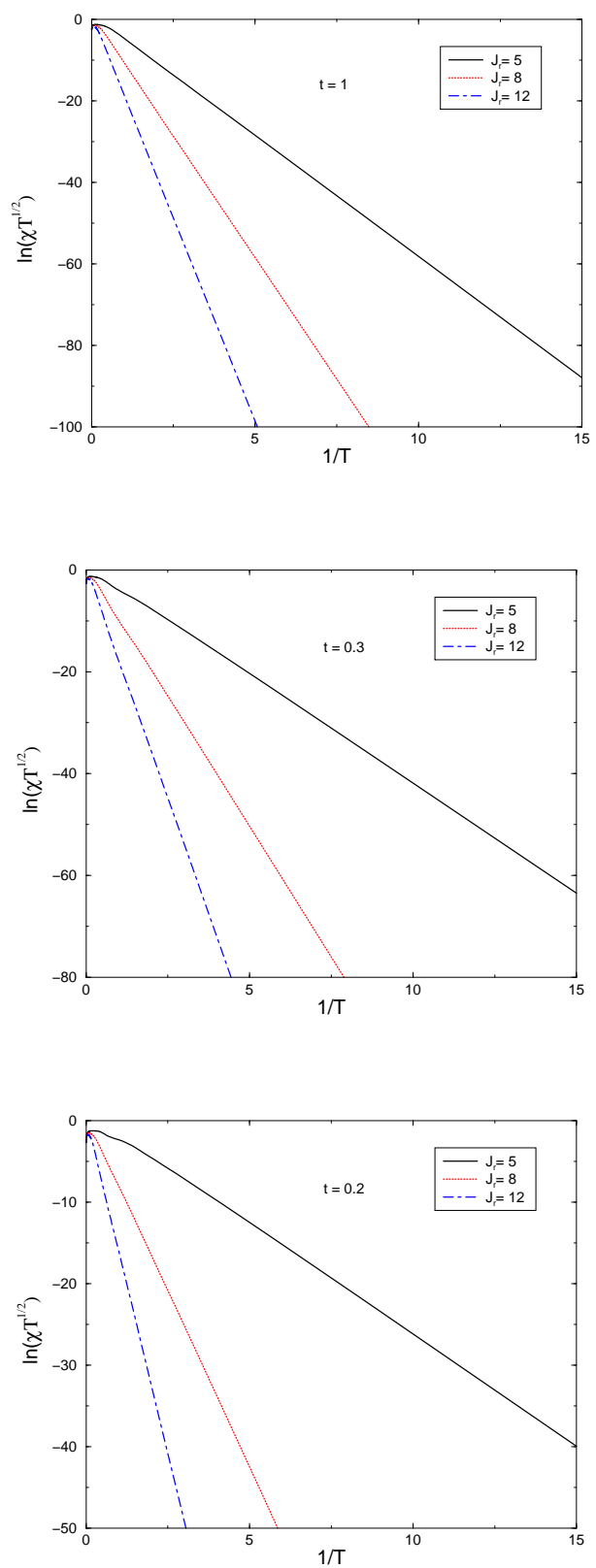

Figure 3: a) The magnetic susceptibility $(\chi)$ as a function of the temperature $(T)$ for different values of the coupling $\left(J_{r}\right)$ and anisotropy $(t)$. (b) A logarithmic plot of the susceptibility $(\chi)$ as a function of the inverse of the temperature $(1 / T)$, from which the spin gap $\left(\Delta^{*}\right)$ can be obtained. 


\begin{tabular}{|c|c|c|c|}
\hline$J_{r}$ & $\mathrm{t}$ & $\Delta$ & $\Delta^{*}$ \\
\hline \multirow{3}{*}{5} & 1.00 & 6.00 & 5.98 \\
\cline { 2 - 4 } & 0.30 & 4.37 & 4.42 \\
\cline { 2 - 4 } & 0.20 & 2.80 & 2.88 \\
\hline \multirow{4}{*}{8} & 1.00 & 12.00 & 11.93 \\
\cline { 2 - 4 } & 0.30 & 10.37 & 10.36 \\
\cline { 2 - 4 } & 0.20 & 8.80 & 8.81 \\
\hline \multirow{4}{*}{12} & 1.00 & 20.00 & 19.88 \\
\cline { 2 - 4 } & 0.30 & 18.37 & 18.32 \\
\cline { 2 - 4 } & 0.20 & 16.80 & 16.76 \\
\hline
\end{tabular}

Table 1: Spin gap $\Delta^{*}$ obtained from the linearization of Eq. (5) and Fig. $3 b$ compared to the analytical result $\Delta$ obtained from the Bethe Ansatz Equations (4) for different values of $J_{r}$ and $t$.

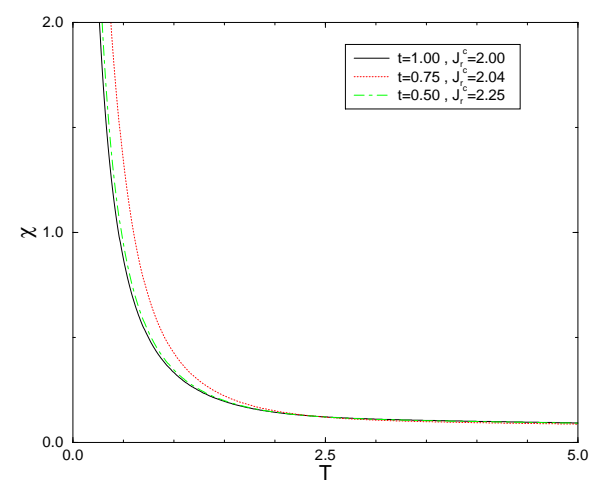

Figure 4: Magnetic susceptibility $(\chi)$ versus temperature $(T)$ for different values of the coupling $J_{r}$ in units of $J_{l}$. These graphs indicate a typical quantum critical behavior.

setting our parameters accordingly we were able to obtain the spin gap for some ladder compounds such as $\mathrm{KCuCl}_{3}\left[1,2, \mathrm{Cu}_{2}\left(\mathrm{C}_{5} \mathrm{H}_{12} \mathrm{~N}_{2}\right)_{2} \mathrm{Cl}_{4}\right.$ [3, 4, 5], $\left(\mathrm{C}_{5} \mathrm{H}_{12} \mathrm{~N}\right)_{2} \mathrm{CuBr}_{4}$ [6] which were previously studied by different authors using different methods. A comparison of our choice of parameters with the experimental data $\Delta^{\exp }[29$ for the above mentioned compounds is depicted in Table 2. For the sake of clarity we present the results on three different compounds separately and refer the reader to the quoted works for more details on the experimental setup.

\section{$3.1 \mathrm{KCuCl}_{3}$}

The magnetic properties of this system were investigated in [1,2] where a non-magnetic ground state and a spin gap in the excitation spectrum were reported. The crystal structure of this compound is moniclinic and the double chain feature of the system arises from two edge-sharing chains of $\mathrm{CuCl}_{6}$ octahedra (see [14 for more details on the geometry of this compound). 


\begin{tabular}{|c|c|c|c|c|c|}
\hline compound & $J_{r}$ & $\mathrm{t}$ & $\Delta$ & $\Delta^{*}$ & $\Delta^{\text {exp }}$ \\
\hline$K C u C l_{3}$ & 4.0 & 0.3254 & 32.00 & 36.56 & 31.10 \\
\hline $\mathrm{Cu}_{2}\left(\mathrm{C}_{5} \mathrm{H}_{12} \mathrm{~N}_{2}\right)_{2} \mathrm{Cl}_{4}$ & 5.5 & 0.2344 & 10.80 & 13.41 & 10.80 \\
\hline$\left(\mathrm{C}_{5} \mathrm{H}_{12} \mathrm{~N}\right)_{2} \mathrm{CuBr} \mathrm{Cu}_{4}$ & 4.0 & 0.3100 & 9.85 & 9.90 & 9.50 \\
\hline
\end{tabular}

Table 2: The values of parameters for different compounds. The experimental data were obtained from Ref. [29].

The experimental data [1] and exact diagonalization results are depicted in Fig. 5.

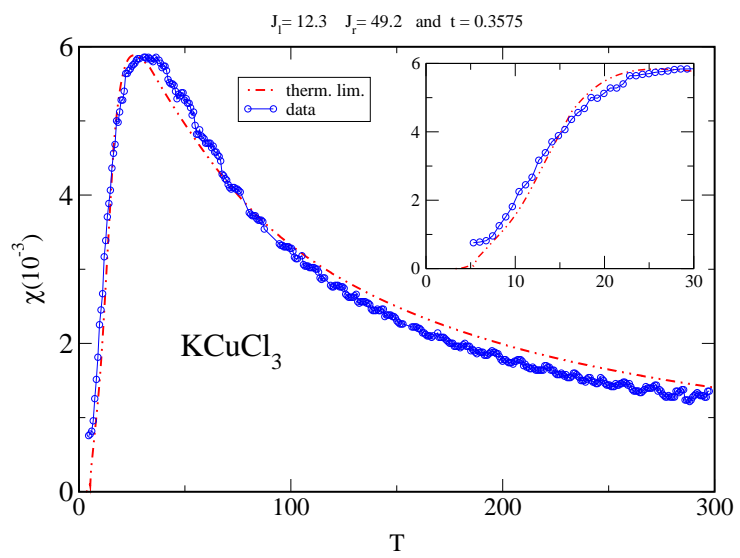

Figure 5: Magnetic susceptibility versus temperature. The full line is the result from extrapolation of finite-size data obtained from exact diagonalization of chains up to 12 spins. Circles are experimental data obtained by Nakamura and Okamoto [1]. The inset shows the low-temperature regime.

The spin gap we used was estimated experimentally to be $\sim 31.10 K[2]$. The full line was obtained using the following exchange couplings: $J_{l}=12.3 \mathrm{~K}, J_{r}=49.2 \mathrm{~K}$ and $t=0.3575$. We observe that the low-temperature regime shows a better agreement between theory and experiment. The deviation in the high-temperature regime might arise from the existence, in the compound, of a ferromagnetic coupling which takes place between the $3 d$ orbitals of $C u^{+2}$ ions mediated by the $C l$ anions [30], a coupling which is not present in our model.

\section{$3.2 \quad \mathrm{Cu}_{2}\left(\mathrm{C}_{5} \mathrm{H}_{12} \mathrm{~N}_{2}\right)_{2} \mathrm{Cl}_{4}$}

This compound have been studied by Chaboussant et al [4, 5, 31. The ladder structure arises from the stacking of $C u-C u$ binuclear units (each $C u$ is to be seen as belonging to one leg). For magnetic fields below $h=7.5 T$ the one-dimensional ground state is a valence-bond singlet structure on each rung and the system is gapped. Above this lower critical field, the first excited state (a triplet) becomes the ground state and the system has a non-zero magnetization. There are basically three different exchange paths between $C u$ 
ions: the intramolecular exchange constant $J_{r}$ between $\mathrm{Cu}-\mathrm{Cu}$ ions takes place through two $C l$ ions while the intermolecular $J_{l}$ involves in-plane unpaired electron densities of $\mathrm{Cu}$ ions of different molecules. The third intermolecular exchange introduces a frustration via next-nearest neighbor interaction and occurs through hydrogen bonds between the $C l$ and $N$ ions. This interaction is however significantly smaller than $J_{r}$ and is taken altogether equal to zero in our model. The low-temperature gap has been determined through susceptibility measurements and NMR techniques to be $\Delta=10.8 \pm 0.6 \mathrm{~K}$ with $J_{l}=2.4 \mathrm{~K}$ and $J_{r}=13.2 \mathrm{~K}[4$. The same gap can be obtained in our model with the choices $J_{l}=2.4 K, J_{r}=13.2 \mathrm{~K}$ and $t=0.2344$. The agreement between the low-temperature experimental data and our results is excellent, as can be seen in Fig. 6, and remains good for the whole temperature range.

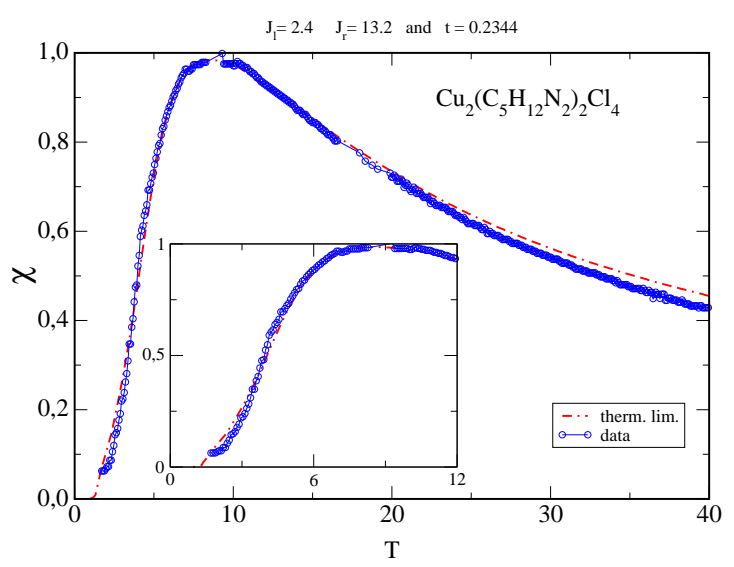

Figure 6: Normalized magnetic susceptibility versus temperature. The full line is the result of exact diagonalization followed by extrapolation of finite-size data. Again we used chains of up to 12 spins. The circles are the experimental data obtained by Chaboussant et al 4]. The inset depicts the low-temperature regime.

\section{$3.3 \quad\left(\mathrm{C}_{5} \mathrm{H}_{12} \mathrm{~N}\right)_{2} \mathrm{CuBr} r_{4}$}

As in the previous compound, here we also have a binuclear $C u-C u$ stacked structure. The intramolecular exchange coupling is mediated through an orbital overlap of $\mathrm{Br}$ ions adjacent to the $C u$ sites. The intermolecular exchange is also mediated by $B r$ ions a bit further apart (8.597 $\AA$ in comparison to intramolecular distances of $6.934 \AA$ ). A diagonal frustration exchange should be present but it is expected to be weak [6]. The exchange couplings used were $J_{l}=4.0 \mathrm{~K}, J_{r}=16.0 \mathrm{~K}$ and $t=0.31 \mathrm{~K}$. Again the fit is better for low temperatures, with an energy gap of $9.8 \mathrm{~K}(9.5 \mathrm{~K}$ experimentally [6] ) and an exponential drop in the magnetic susceptibility. The data is depicted in Fig. 7. 


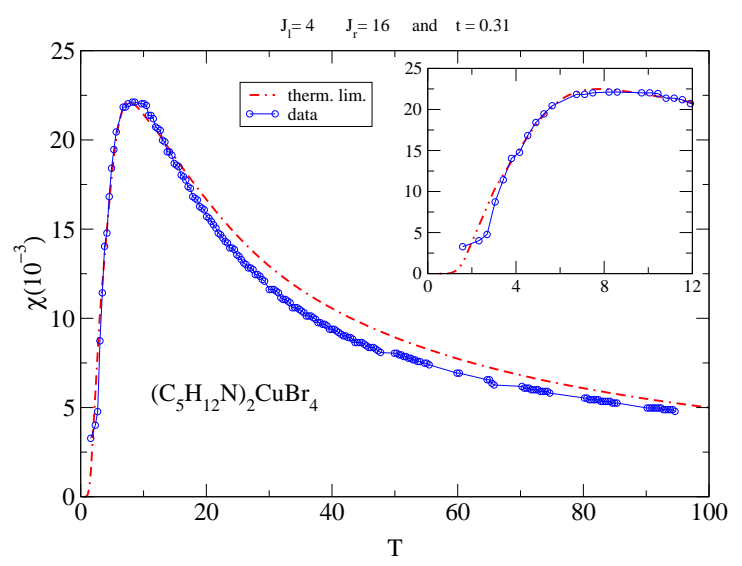

Figure 7: Magnetic susceptibility versus temperature. Circles represent experimental data obtained by Watson et. al. 6]. The solid line is the result obtained via exact diagonalization as explained in the previous captions. The inset depicts the low-temperature regime.

\section{Summary}

We presented a numerical analysis of thermodynamic properties of an integrable anisotropic spin ladder model which has, besides the rung and leg couplings, an extra free anisotropy parameter. From the Bethe Ansatz equations as well as the exact expression for the energy spectrum [23] we showed that the model exhibits an anisotropy- dependent spin gap. The critical line and phase diagram were obtained. The magnetic susceptibility was investigated and good agreement was found between the gap obtained from the Bethe ansatz equations and the gap obtained from the magnetic susceptibility. A connection with some strong coupling compounds was discussed and our results reproduce well experimental data for these compounds in strong coupling limit. The anisotropy parameter in our model has no obvious physical interpretation. However, cyclic four-spin exchange terms are known to affect the excitation spectrum of spin ladders and the importance of this type of interaction for appropriately describing the properties of strong-coupling ladders has also been established [32]. As shown in this work the anisotropy parameter allows us to tune our model to different physical systems of interest, thus presenting a unified scenario for strong-coupling ladders.

This work has been financially supported by the brazilian federal agency CNPq (APT, $\mathrm{AF}$ and ALM) and the state agency FAPESP (ALM).

\section{References}

[1] T. Nakamura and K. Okamoto, Phys. Rev. B58, 2411 (1998)

[2] H. Tanaka, K. Takatsu, W. Shiramura and T. Ono, J. Phys. Soc. Jap..65, 1945 (1996)

[3] C. A. Hayward, D. Poilblanc and L. P. Lèvy, Phys. Rev.B54, R12649 (1996) 
[4] G. Chaboussant et. al., Phys. Rev. Lett. 19, 925 (1997); J. de Gier and M.T. Batchelor, Phys. Rev. B62,

[5] G. Chaboussant et. al., Phys. Rev. Lett. 80, 2713 (1998)

[6] B. C. Watson et. al., Phys. Rev. Lett. 86, 5168 (2001)

[7] J. B. Bednorz and K. A. Müller, Z. Phys. B 64, 189 (1986)

[8] E. Dagotto, Rep. Prog. Phys. 62, 1525 (1999)

[9] H. Bethe, Z. Phys. 71, 205 (1931)

[10] E. Dagotto and T. M. Rice, Science 271, 618 (1996)

[11] T. M. Rice, S. Gopalan and M. Sigrist, Europhys. Lett.23, 445 (1993)

[12] S. R. White, R. M. Noack and D. J. Scalapino, Phys. Rev.Lett. 73, 886 (1994)

[13] S. Albeverio, S.-M. Fei and Y. Wang, Europhys. Lett. 47, 364 (1999)

[14] M.T. Batchelor and M. Maslen, J. Phys. A: Math. Gen. 32, L377 (1999); J. Phys A 33, $443(2000)$

[15] M.T. Batchelor, J. de Gier, J. Links and M. Maslen, J. Phys. A 33, L97 (2000)

[16] J. de Gier, M.T. Batchelor and M. Maslen, Phys. Rev. B62, 15196 (2000)

[17] J. de Gier, M.T. Batchelor and M. Maslen, Phys. Rev. B62, 15196 (2000)

[18] J. Links and A. Foerster, Phys. Rev. B62, 65 (2000)

[19] A. Kundu, J. Math Phys 41, 721 (2000)

[20] A. Foerster, K.A. Hibberd, J.R. Links and I. Roditi, J. Phys. A: Math. Gen. 34, L25 (2001)

[21] J. Ambjorn, D. Arnaudon, A. Sedrakyan, T. Sedrakyan and P. Sorba, J. Phys. A.: Math. Gen. 34, 5887 (2001)

[22] H. Frahm and A. Kundu, J. Phys. C11, L557 (1999)

[23] A. P. Tonel, A. Foerster, J. Links and A. L. Malvezzi, Phys. Rev..B 64, 054420-1 (2001)

[24] A. A. Nersesyan and A. M. Tsvelik, Phys. Rev. Lett. 78, 3939 (1997)

[25] Y. Wang, Phys. Rev. B 60, 9236 (1999)

[26] J. de Gier and M.T. Batchelor, Phys. Rev. B62, R3584 (2000)

[27] R. Bulirsh and J. Stoer, Num. Math.6, 413 (1994) 
[28] M. Troyer, H. Tsunetsugu and D. Würtz, Phys. Rev. B50, 13515 (1994)

[29] E. Dagotto and T.M. Rice, Science 271, 618 (1996)

[30] J. Kanamori, J.Phys. Chem. Solids 10, 87 (1959)

[31] G. Chaboussant, P. A. Crowell, L. P. Levy, O. Piovesana, A. Madouri and D. Mailly, Phys. Rev.B55, 3046 (1998)

[32] T. S. Nunner et. al., Phys. Rev. B66, 180404(R) (2002); N. Haga and S. Suga, Phys. Rev. B66, 132415 (2002). 